\title{
Peer Community In
Paleontology
}

$\because$

paleo.peercommunityin.org

Open Access

\section{Vomers aren't so different in crown group birds when considering allometric effects}

\author{
Andrew Farke ${ }^{1}$ \\ ${ }^{1}$ Raymond M. Alf Museum of Paleontology at The Webb Schools - Claremont, CA, USA
}

Cite as: Farke $A$ (2021). Vomers aren't so different in crown group birds when considering allometric effects. Peer Community In Paleontology, 100007. DOI: 10.24072/pci.paleo.100007

Published: 14 April 2021

Based on reviews by: Sergio Martínez Nebreda and Roland Sookias

\author{
A recommendation of \\ Plateau O and Foth C (2021). The impact of allometry on vomer shape and its implications for the \\ taxonomy and cranial kinesis of crown-group birds. bioRxiv 184101, ver. 3 peer-reviewed by PCI \\ Paleo. DOI: 10.1101/2020.07.02.184101
}

Today's birds are divided into two deeply divergent and historically well-documented groups: Palaeognathae and Neognathae. Palaeognaths include both the flight-capable tinamous as well as the flightless ratites (ostriches, rheas, kiwis, cassowaries, and kin). Neognaths include all other modern birds, ranging from sparrows to penguins to hummingbirds. The clade names refer to the anatomy of the palate, with the "old jaws" (palaeognaths) originally thought to more closely resemble an ancestral reptilian condition and the "new jaws" (neognaths) showing a uniquely modified bony configuration. This particularly manifests in the pterygoid-palatine complex (PPC) in the palate, formed from pairs of pterygoids and palatines alongside a single midline vomer. In palaeognaths, the vomer is comparatively large and the pterygoid and palatine are relatively tightly connected. The PPC is more mobile in neognaths, with a variably shaped vomer, which is sometimes even absent. Although both groups of birds show cranial kinesis, neognaths exhibit a much more pronounced degree of kinesis versus palaeognaths, due in part to the tighter nature of the palaeognath pterygoid/palatine interfaces.

A previous paper (Hu et al., 2019) used 3D geometric morphometrics to compare the shape of the vomer across neognaths and palaeognaths. Among other findings, this work suggested that each clade had a distinct vomer morphology, with palaeognaths more similar to the ancestral condition (i.e., that of non-avian dinosaurs). This observation was extended to support inferences of limited vs. less limited cranial kinesis in various extinct species, based in part on observations of vomer shape. A new preprint by Plateau and Foth (2021) presents a reanalysis of Hu et al.'s data, specifically focusing on allometric effects. In short, the new analysis looks at how size correlates (or doesn't correlate) with vomer shape.

Plateau and Foth (2021) found that when size effects are included, differences between palaeognaths and neognaths are less than the "raw" (uncorrected) shape data suggest. It is much harder to tell bird groups apart! Certainly, there are still some general differences, but some separations in morphospace close up when allometry-the interrelationship between shape and size-is considered. Plateau and 
Foth (2021) use this finding to suggest that 1) vomer shape alone is not a completely reliable proxy for inferring the phylogenetic affinities of a particular bird; and 2) the vomer is only one small component of the cranial kinetic system, and thus its shape is of limited utility for inferring cranial kinesis capabilities when considered independently from the rest of the relevant skull bones.

\section{References}

Hu H, Sansalone G, Wroe S, McDonald PG, O'Connor JK, Li Z, Xu X, and Zhou Z (2019). Evolution of the vomer and its implications for cranial kinesis in Paraves. Proceedings of the National Academy of Sciences 116, 19571-19578. doi: 10.1073/pnas.1907754116.

Plateau $O$ and Foth C (2021). The impact of allometry on vomer shape and its implications for the taxonomy and cranial kinesis of crown-group birds. bioRxiv 184101, ver. 3 peer-reviewed by PCI Paleo. doi: 10.1101/2020.07.02.184101.

\section{Appendix}

Reviews by Sergio Martínez Nebreda and Roland Sookias, DOI: 10.24072/pci.paleo.100007. 